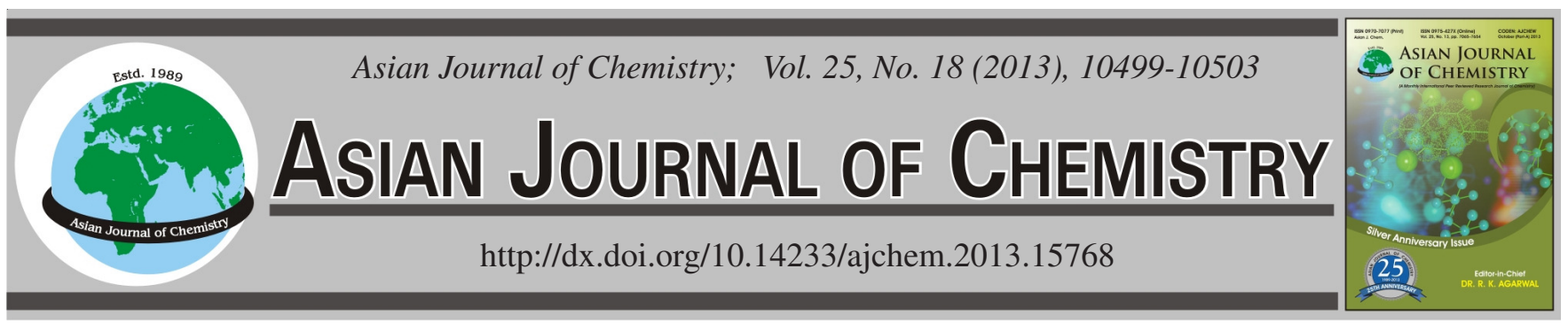

\title{
Dye-Surfactant Interaction: Role of Solvent
}

\author{
Ziya Ahmad Khan ${ }^{*}$ and Abdullah S. Al-Bogami
}

Department of Chemistry, Faculty of Science, North Jeddah Centre, King AbdulAziz University, P.O. Box 80203, Jeddah 21589, Saudi Arabia

*Corresponding author: E-mail: ziyaakhan@gmail.com

\begin{abstract}
Surfactant-dye associations are important not only in dyeing process but also in dye separation processes such as cloud point extraction or micellar enhanced ultrafiltration. Many techniques were used for qualitative and quantitative description of dye-surfactant interactions, i.e., potentiometry, conductometry, or ion selective electrodes and spectrophotometry. In the presence of surfactant new bands in the electronic absorption spectra of many dyes can appear and the stronger the mutual interaction between dye and surfactant the greater change is observed. The changes of visible absorption spectra of dye in the presence of surfactant at different concentrations result from equilibrium between surfactant monomers, micelles, dye aggregates, dye-surfactant premicellar complex and dye particles incorporated into the micelles. The solvents also plays an important role, as its polarity affects the interaction between the surfactants and dyes. Therefore, the understanding of those interactions enables the prediction of characteristic behaviour of the dye-surfactant mixture in the aqueous and mixed solvent systems. In this paper, we have studied the effect of solvents (especially low per cent) on the interaction of methyl orange with cationic surfactant in presence of various solvents, such as, ethylene glycol, dimethylformamide, dioxane, formamide and acetonitrile.
\end{abstract}

Key Words: Surfactant, Dye, Solvent, UV-visible spectrophotometer.

(1)

\section{INTRODUCTION}

Surfactants have a tendency to accumulate at the air-liquid interface and thus they can change the properties of interface. In addition, because of their amphipathic structures, they can congregate to form a stabilized entity, called micelle, after the attainment of critical micelle concentration $(\mathrm{CMC})^{1}$. One of the most outstanding properties of surfactants are their ability to solubilize molecules which otherwise would not be soluble in water. Many dyes, polynuclear hydrocarbons and proteins are stabilized in presence of micelles through displaying marked spectral and colour changes. The interaction between dyes and surfactants are subject of numerous investigations ${ }^{2-8,9,10}$. Among the different industrial applications, surfactants are used in dye industry as they can wet ${ }^{11}$ and help in the dispersion of dyes, especially which are poorly soluble.

Surfactants are used to assist in dyeing, by wetting and leveling or dispersing dyes of low solubility. Their interactions with dyes play an important role in achieving level of dyeing $^{12,13}$.

Dyes exhibit significant spectral changes in micellar media $^{14}$. It has been suggested that owing to electrostatic interactions, the ionic dyes can form molecular complexes with oppositely charged micelles. The stability of such complexes depends on the interactions between the dye and the surfactant molecule. Therefore, the understanding of those interactions enables the prediction of characteristics behaviour of the dyesurfactant mixture in the solution.

The spectral changes of a dye observed in the presence of varying amount of surfactants are consistent with sequential equilibria involving surfactant monomers, micelles, dye aggregates, premicellar dye/surfactant complex and dye incorporated to micelle $\mathrm{e}^{15-18}$. Extensive research carried out recently has confirmed the ability of surfactants to affect the electronic absorption spectra of solutions of many dyes, viz., triphenylmethane ${ }^{19,20}, \mathrm{azo}^{21}$ and phenothazine ${ }^{22}$. Two types of interaction between dye and surfactant may be observed depending on the chemical structure of both dye and surfactant: at concentrations below the critical micelle concentration, decrease in the absorbance with appearance of a new band indicates formation of a complex between dye and surfactant and at high micelle concentrations, increased extinction coefficient with a red shift which indicates incorporation of dye to micelle ${ }^{15,18}$. The role of solvents plays an important role as the solvent polarity affects the interaction between the surfactants and dyes. Therefore, the understanding of those interactions enables the prediction of characteristic behaviour of the dyesurfactant mixture in the aqueous and mixed solvent systems. 


\section{EXPERIMENTAL}

Methyl orange (MO) obtained from Pacegrove limited (UK) used as received. Cetyltrimethylammonium bromide (CTAB , $\geq 98 \%$ ) was purchased from Sigma. Ethylene glycol (EG), 1,4-dioxane (DO), acetonitrile (AN), dimethyl formamide (DMF) and formamide (FM) were of AR grade and used as such. The water used to prepare the sample solutions was demineralized and double distilled in an all-glass distillation apparatus. The specific conductivity of this water was in the range (1-2) $10^{-6} \mathrm{~S} \mathrm{~cm}^{-1}$.

The electronic absorption spectra were recorded on $\mathrm{T} 70+$ UV-visible spectrophotometer (PG, Instruments, U.K.) using a matched pair of quartz cuvettes of $1 \mathrm{~cm}$ path-length.

Stock solution of methyl orange and CTAB prepared. Then the sample solutions were prepared by taking requisite amounts in the different solvents $(\% \mathrm{v} / \mathrm{v})$, keeping the surfactant and dye concentration constant. After mixing, the sample solutions were kept overnight for equilibration.

\section{RESULTS AND DISCUSSION}

The electronic absorption spectra of solution of many dyes affect by the presence of organized media, such as, micelles. The position of the long-wavelength absorption band and the intensity of dyes are sensitive to the medium; therefore they can be used as solvatochromatic micro polarity reporter molecules. As seen as in Fig. 1, the anionic dye methyl orange in aqueous solution exhibits a maximum absorption band at $465 \mathrm{~nm}$.

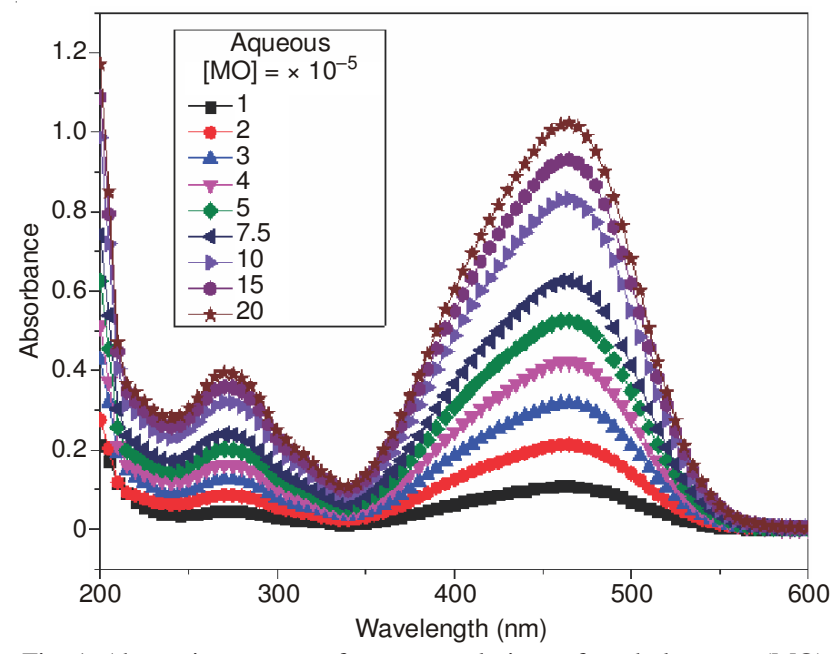

Fig. 1. Absorption spectra of aqueous solutions of methyl orange (MO)

The interaction of methyl orange with CTAB in presence of various solvents results in decrease the maximum absorption spectra and, in some cases, the appearance of a new absorption band on the spectrum of the dye. Only one of them, the spectrum of dye in the presence of $0.2 \times 10^{-3} \mathrm{M} \mathrm{CTAB}$ concentration, is shown in Fig. 2. These spectral changes can be attributed to the formation of a complex between dye and surfactant molecules $^{23}$. The changes in the absorption spectrum of dye with increasing surfactant concentrations (premicellar to micellar form) with different per cent of solvents is observed (all the spectra are not shown).

Fig. 3 shows the absorbance changes of $2.0 \times 10^{-5} \mathrm{M}$ methyl orange in presence of $0.2 \mathrm{mM}$ CTAB solution with increasing solvent per cent at only $465 \mathrm{~nm}$ wavelength.
As shown in Fig. 3, the absorbance of dye showed a decrease in the intensity at $465 \mathrm{mM}$, with increasing per cent of solvents in a fixed surfactants concentration, reaching a minimum value and then a sharp increase showed in intensity of the absorption band.

Effect of solvent: From the Fig. 3, it is clear that for the premicellar region $(0.2 \mathrm{mM}$ and $0.6 \mathrm{mM}$ CTAB $)$, a sharp decrease in absorbance up to $15 \%$ solvents. This suggests that there is a strong interaction between dye and surfactant molecule. For 0.2 and $0.6 \mathrm{mM}$ concentration, CTAB molecule remains as monomer. The negatively charges methyl orange molecule interact with monomer of cationic CTAB strongly to form dye-surfactant complex, hence decrease in absorbance occurs. However, further increase in per cent of solvents, absorbance of methyl orange increases, except ethylene glycol for $0.2 \mathrm{mM} \mathrm{CTAB}$ and formamide for $0.6 \mathrm{mM} \mathrm{CTAB}$. This can be understood by the fact that with increasing per cent of solvents affects the polarity of the solution and also the solventsolvent interaction affects interaction between dye and surfactant. Formamide and ethylene glycol are protic solvent forming a hydrogen bonding between water and these solvents.

Fig. 3 showed that with increasing concentration of CTAB, the decrease in absorbance of methyl orange above the $1 \mathrm{mM}$ CTAB concentration is due to the increase in the amount of solubilized methyl orange in micelles. With increasing surfactant concentration the absorbance values reached the limiting value. This case indicates that all dye molecules are compartmentalized in to micelles ${ }^{5,17}$ i.e. in the certain surfactant concentration the solubilization of dye in the micelles is practically completed and addition of surfactants failed to cause any spectral change. The decrease in absorbance of methyl orange is due to some sort of association or complex formation between dye and surfactant monomers. The results indicate that hydrophobicity of alkyl chain plays an important role in complex formation. The formation of the dye-surfactant complex is a consequence of mutual influences of long-range and shortrange interactions. Ionic surfactants as well as methyl orange possess a strong polar group. In aqueous solution they dissociate into ions and therefore electrostatic attractive interaction can appear between head group of dye and head groups of surfactant. As the electrostatic attraction between the oppositely charged ions bring them in to close proximity, short-range dispersive forces or hydrophobic interactions must align the hydrocarbon chain of the surfactant with the hydrophobic moiety of the dye. Presence of solvents, change the environment of the solution for the dye and surfactant interaction. It is also known that both kinds of attractive forces are present among oppositely charged dyes and surfactant, long-range electrostatic coulombic forces and short-range dispersive and hydrophobic forces which act cooperatively. The non-polar part of the surfactant is an aliphatic chain so it is very likely that hydrophobic interaction will appear in the process. While surfactant concentration increases, more micelle forms. First, while surfactant concentration was increased close to CMC concentration absorbance reduced. After CMC value, an increase in absorbance is observed. This behaviour can be explained as the monomeric, dimeric and trimeric conversion. Methyl orange-CTAB association process can be assumed to take place in two principal steps 

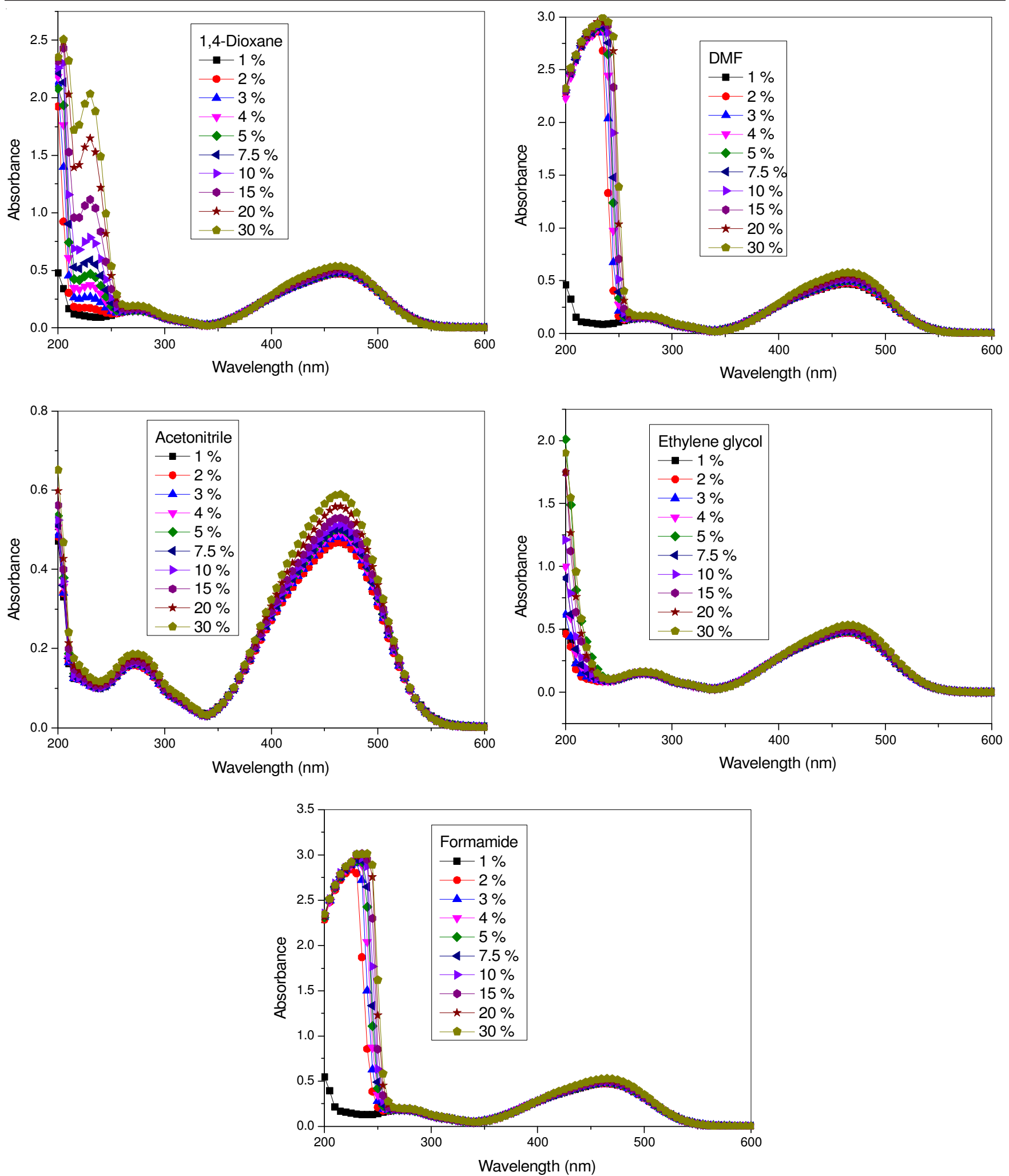

Fig. 2. Absorption spectra of solutions of $[\mathrm{MO}]=2 \times 10^{-5} \mathrm{M}$ in $[\mathrm{CTAB}]=0.2 \times 10^{-3} \mathrm{M}$ with various percent of solvents

and consistent with previous observation for many dye-surfactant systems ${ }^{18,24}$. In the first step, at very low surfactant concentrations, far below their CMC, the formation of dyesurfactant complex species is mainly governed by electrostatic long-range and disperse short-range attractive force between dye and surfactant ions. In the second step, at the increasing surfactant concentration, dye and micelles are exist in the solution. Near and just below the CMC, dye-surfactant aggregates, reorganize into premicelles with a monomeric dye content resulting in an increase in the absorbance of the dye in this premicellar region (just below the CMC). On further addition of surfactant, micelles occur, the absorbance reaches its limiting value and all dye molecules are accommodated into normal micelles as monomeric molecules. 

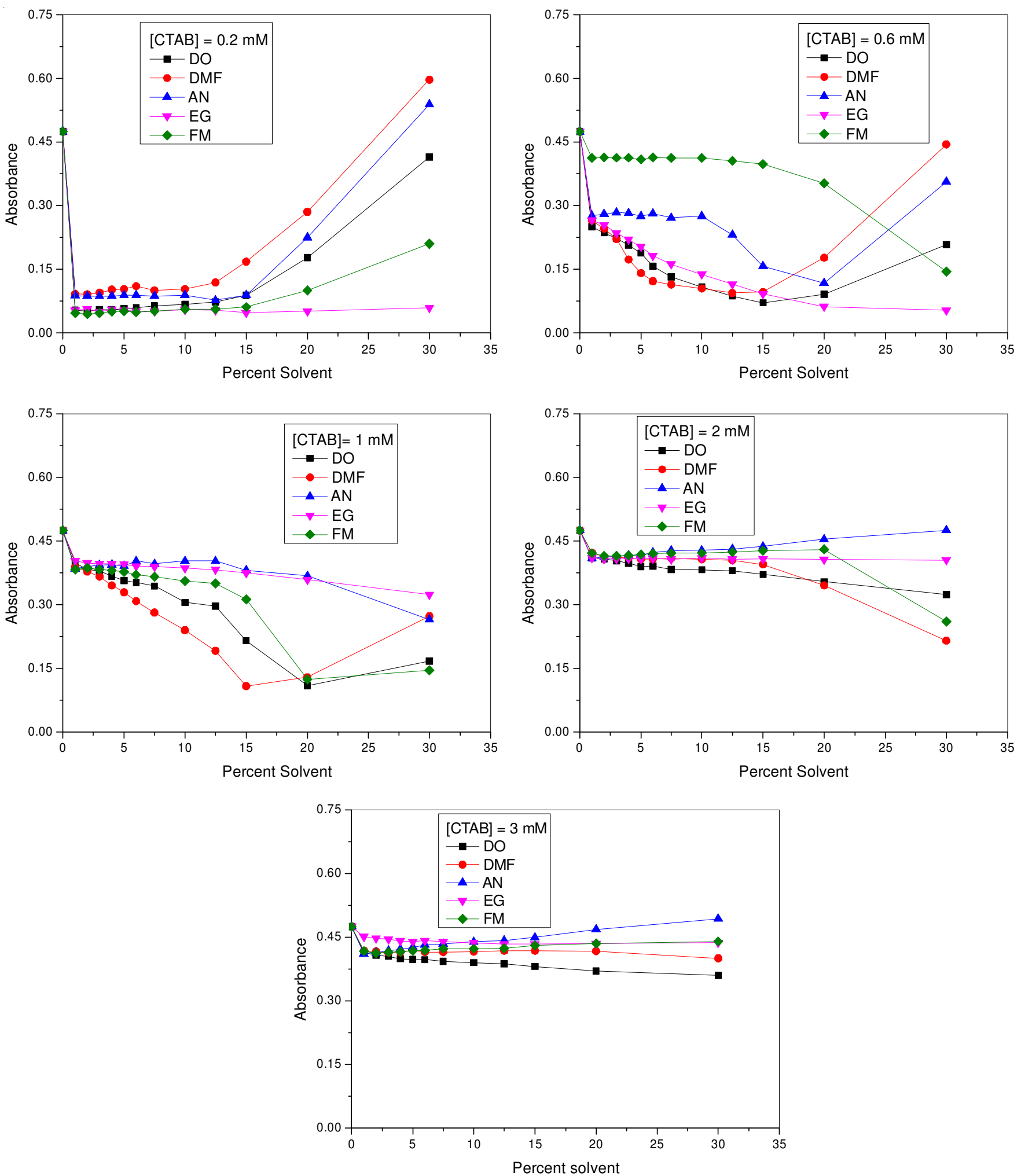

Fig. 3. Changes of maximum absorbance of dye with increasing solvent per cent at $465 \mathrm{~nm}$

\section{Conclusion}

In this article, the interaction of cationic cetyltrimethylammonium bromide surfactants with methyl orange in presence of solvents was analyzed by UV-visible absorption spectrophotometer. It was seen that interaction was occurred even at low per cent of solvent and continued until the dye becomes fully saturated with surfactant. At low per cent of solvent, the decrease in spectral values indicated dye-surfactant complex formation. This was resulted from the strong interactions between dye and head groups of surfactant. This study shows that for the interaction between cationic surfactants and anionic dye the solvents nature and its ability to form ion-pair with water and electrostatic and hydrophobic forces were important. 


\section{ACKNOWLEDGEMENTS}

This paper was funded by the Deanship of Scientific Research (DSR), King Abdulaziz University, Jeddah, under grant No. (6-965-D1432).

\section{REFERENCES}

1. S.P. Moulik, Curr. Sci., 71, 368 (1996).

2. R.K. Dutta and S.N. Bhat, Can. J. Chem., 71, 1785 (1993).

3. D. Pramanick and D. Mukherjee, J. Colloid Interf. Sci., 157, 131 (1993).

4. G. Nowicka and W. Nowicki, J. Chem. Phys., 91, 247 (1994).

5. S.S. Shah, M.S. Khan, H. Ullah and M.A. Awan, J. Colloid Interf. Sci., 186, 382 (1997).

6. K.K. Karukstis, D.A. Savin, C.T. Loftus and N.D. D'Angelo, J. Colloid Interf. Sci., 203, 157 (1998).

7. M. Sarkar and S. Poddar, Spectrochim. Acta A, 55, 1737 (1999)

8. Z. Yuan, M. Zhu and S. Han, Anal. Chim. Acta, 389, 291 (1999).

9. S. Gokturk and M. Tuncay, Spectrochim. Acta A, 59, 1857 (2003).

10. M. Chakraborty and A.K. Panda, Spectrochim. Acta A, 81, 458 (2011).
11. S.M. Ghoreishi, M. Behpour and A.G. Farsani, Dyes Pigments, 74, 585 (2007).

12. Y. Yang and C.M. Ladisch, Textile Res. J., 63, 283 (1993).

13. P.F. Tavcer and J. Span, Textile Res. J., 69, 278 (1999).

14. E. Barni, P. Savarino and G. Vicardi, Acc. Chem. Res., 24, 98 (1991).

15. M.J. Minch and S.S. Shah, J. Org. Chem., 44, 3252 (1979).

16. M.A. Awan and S.S. Shah, Colloids Surf. A, 122, 97 (1997).

17. S.S. Shah, G.M. Laghari and K. Naeem, Thin Solid Films, 346, 145 (1999).

18. M. Sarkar and S. Poddar, J. Colloid Interf. Sci., 221, 181 (2000).

19. E. Colichman, J. Am. Chem. Soc., 73, 3385 (1951).

20. J. Rosendorfova and L. Cermakova, Talanta, 27, 705 (1980).

21. S.B. Savvin, R.K. Chernova and S.N. Shtykov, Zh. Anal. Khim., 33, 88 (1978).

22. I. Nemcova and M. Duskeva, Collect. Czech. Chem. Commun., 51, 296 (1992).

23. D.M. Stevensen, D.G. Duff and D.J. Kirkwood, J. Soc. Dyers Colour, 97, 13 (1981).

24. S. Yamamoto, S. Kobashi, K. Tsutsui and Y. Sueishi, Spectrochim. Acta, 66, 302 (2007). 